\title{
Validated solution of ODEs with parametric uncertainties
}

\author{
Youdong Lin ${ }^{\mathrm{a}}$ and Mark A. Stadtherr ${ }^{\mathrm{a}}$ \\ aDepartment of Chemical and Biomolecular Engineering, University of Notre Dame, \\ Notre Dame, IN 46556 USA
}

\begin{abstract}
We introduce a new methodology for computing a validated enclosure of all solutions of an ODE system with interval-valued parameters and/or initial values. The method is based on a traditional interval approach, but involves a novel use of Taylor models to address the dependency problem of interval arithmetic. Numerical results on a bioreactor kinetics problem, with uncertain initial biomass concentration and uncertain kinetic parameters, demonstrate that this approach provides a very effective way to obtain an enclosure of all possible solutions to a parametric ODE system under uncertain conditions.
\end{abstract}

Keywords: ODE, Validated computing, Parametric uncertainty, Bioreactor kinetics

\section{Introduction}

Initial value problems (IVPs) for ordinary differential equations (ODEs) arise naturally in many applications in process engineering. However, it is often the case that the parameters and/or the initial values are not known with certainty. One common way to represent the uncertainties in such parametric ODEs is to treat the parameters and/or initial values as intervals. Thus, consider the problem of determining a validated enclosure of all solutions of the parametric autonomous IVP,

$\boldsymbol{y}^{\prime}(t)=\boldsymbol{f}(\boldsymbol{y}, \boldsymbol{\theta}), \quad \boldsymbol{y}\left(t_{0}\right)=\boldsymbol{y}_{0} \in \boldsymbol{Y}_{0}, \quad \boldsymbol{\theta} \in \boldsymbol{\Theta}$,

where $t \in\left[t_{0}, t_{m}\right]$ for some $t_{m}>t_{0}$. Here $\boldsymbol{\theta}$ is a $p$-dimensional vector of time-invariant parameters, $\boldsymbol{y}$ is the $n$-dimensional vector of state variables, and $\boldsymbol{y}_{0}$ is the $n$-dimensional vector of initial values. The interval vectors $\boldsymbol{\Theta}$ and $\boldsymbol{Y}_{0}$ represent enclosures of the uncertainties in $\boldsymbol{\theta}$ and $\boldsymbol{y}_{0}$, respectively.

Interval methods [1] (also called validated or verified methods) for ODEs can not only determine a guaranteed error bound on the true solution, but can also verify that a unique solution to the problem exists. Traditional interval methods usually consist of two processes applied at each integration step [1]. In the first process, existence and uniqueness of the solution are proven using the Picard-Lindelöf operator and the Banach fixed point theorem, and a rough enclosure of the solution is computed. In the second process, a tighter enclosure of the solution is computed. In general, both processes are realized by applying interval Taylor series (ITS) expansions with respect to time, and 
using automatic differentiation to obtain the Taylor coefficients. An excellent review of the traditional interval methods has been given by Nedialkov et al. [2]. For addressing this problem, there are various packages available, including AWA [3], VNODE [4] and COSY VI [5], all of which consider uncertainties in initial values only.

In the work described here, we will describe a method for efficiently determining validated solutions of ODEs with parametric uncertainties. The method makes use, in a novel way, of the Taylor model approach that Makino and Berz [6] used to deal with the dependence problem in interval arithmetic, and which they applied in COSY VI [5].

\section{Taylor Models}

Makino and Berz [6,7] have described a remainder differential algebra (RDA) approach for bounding function ranges and controlling the dependency problem of interval arithmetic. This method expresses a function by a model consisting of a Taylor polynomial, usually a truncated Taylor series, and an interval Taylor remainder bound. Consider a function $f: \boldsymbol{x} \in \boldsymbol{X} \subset \mathbb{R}^{m} \rightarrow \mathbb{R}$ that is $(q+1)$ times partially differentiable on $\boldsymbol{X}$. Based on the Taylor expansion about the point $\boldsymbol{x}_{0} \in \boldsymbol{X}$, the Taylor model of $f(\boldsymbol{x})$ then consists of a $q$-th order polynomial function in $\left(\boldsymbol{x}-\boldsymbol{x}_{0}\right), p_{f}$,

$p_{f}=\sum_{i=0}^{q} \frac{1}{i !}\left[\left(\boldsymbol{x}-\boldsymbol{x}_{0}\right) \cdot \nabla\right]^{i} f\left(\boldsymbol{x}_{0}\right)$

and an interval remainder bound $R_{f}$, evaluated here in interval arithmetic,

$R_{f}=\frac{1}{(q+1) !}\left[\left(\boldsymbol{X}-\boldsymbol{x}_{0}\right) \cdot \nabla\right]^{q+1} F\left[\boldsymbol{x}_{0}+\left(\boldsymbol{X}-\boldsymbol{x}_{0}\right) \Xi\right]$

and is denoted $T_{f}=\left(p_{f}, R_{f}\right)$, where $\Xi=[0,1]$, and $[\boldsymbol{g} \cdot \nabla]^{k}$ is the operator

$[\boldsymbol{g} \cdot \nabla]^{k}=\sum_{\substack{j_{1}+\cdots+j_{m}=k \\ 0 \leq j_{1}, \cdots, j_{m} \leq k}} \frac{k !}{j_{1} ! \cdots j_{m} !} g_{1}^{j_{1}} \cdots g_{m}^{j_{m}} \frac{\partial^{k}}{\partial x_{1}^{j_{1}} \cdots \partial x_{m}^{j_{m}}}$

Arithmetic operations with Taylor models can be done using the RDA operations described by Makino and Berz [6,8], which include addition, multiplication, reciprocal, and intrinsic functions. Therefore, for any function representable in a computer environment, it is possible to compute a Taylor model using RDA operations by simple operator overloading. In performing RDA operations, only the coefficients of $p_{f}$ are stored and operated on. Computation of bounds on $T_{f}$ over $\boldsymbol{X}$ is denoted by $B\left(T_{f}\right)$. It has been shown that, compared to other rigorous bounding methods, the Taylor model can be used to obtain sharper bounds for modest to complicated functional dependencies $[6,7,9]$.

\section{Validated Solver for Parametric ODEs}

The method proposed here uses the traditional two-phase approach, but makes use of Taylor models to deal with the dependency problem arising due to the uncertain quantities (parameters and initial values). In phase 1 , the goal is to find a step size $h_{j}=t_{j+1}-t_{j}>0$, 


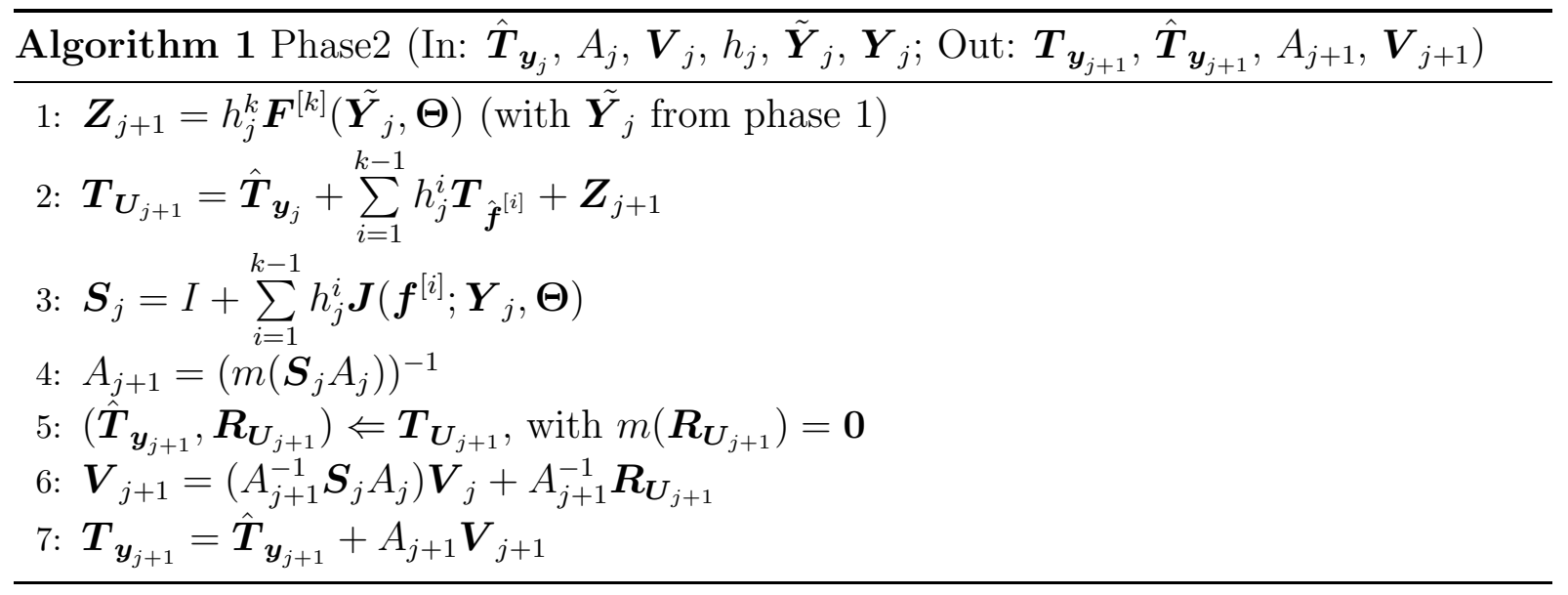

and a rough enclosure $\tilde{\boldsymbol{Y}}_{j}$ of the solution such that existence and uniqueness of the solution can be verified. We apply the traditional interval method to the parametric ODEs by using an ITS with respect to time; that is, for $\boldsymbol{Y}_{j} \subseteq \tilde{\boldsymbol{Y}}_{j}^{0}, h_{j}$ and $\tilde{\boldsymbol{Y}}_{j}$ are determined such that

$\tilde{\boldsymbol{Y}}_{j}=\sum_{i=0}^{k-1}\left[0, h_{j}\right]^{i} \boldsymbol{F}^{[i]}\left(\boldsymbol{Y}_{j}, \boldsymbol{\Theta}\right)+\left[0, h_{j}\right]^{k} \boldsymbol{F}^{[k]}\left(\tilde{\boldsymbol{Y}}_{j}^{0}, \boldsymbol{\Theta}\right) \subseteq \tilde{\boldsymbol{Y}}_{j}^{0}$

where $\boldsymbol{F}^{[i]}$ is the interval extension of $\boldsymbol{f}^{[i]}$, the $i$-th Taylor coefficient in terms of $\boldsymbol{y}^{\prime}(t)=$ $\boldsymbol{f}(\boldsymbol{y}, \boldsymbol{\theta})$. Eq. (5) demonstrates that there exists a unique solution $\boldsymbol{y}\left(t ; t_{j}, \boldsymbol{y}_{j}, \boldsymbol{\theta}\right) \in \tilde{\boldsymbol{Y}}_{j}$ for all $t \in\left[t_{j}, t_{j+1}\right]$, any $\boldsymbol{y}_{j} \in \boldsymbol{Y}_{j}$, and any $\boldsymbol{\theta} \in \boldsymbol{\Theta}$.

In phase 2, we compute a tighter enclosure $\boldsymbol{Y}_{j+1} \subseteq \tilde{\boldsymbol{Y}}_{j}$ such that $\boldsymbol{y}\left(t_{j+1} ; t_{0}, \boldsymbol{Y}_{0}, \boldsymbol{\Theta}\right) \subseteq$ $\boldsymbol{Y}_{j+1}$. This will be done by using an ITS approach to compute a Taylor model $\boldsymbol{T}_{\boldsymbol{y}_{j+1}}$ of $\boldsymbol{y}_{j+1}$ in terms of the uncertain quantities (initial values and parameters), and then obtaining the enclosure $\boldsymbol{Y}_{j+1}=B\left(\boldsymbol{T}_{\boldsymbol{y}_{j+1}}\right)$. For the Taylor model computations, we begin by representing the interval initial values $\boldsymbol{y}_{0} \in \boldsymbol{Y}_{0}$ by a Taylor model with components

$T_{y_{0 i}}=\left(m\left(Y_{0 i}\right)+\left(y_{0 i}-m\left(Y_{0 i}\right)\right),[0,0]\right), \quad i=1, \cdots, n$,

and the interval parameters $\boldsymbol{\theta} \in \boldsymbol{\Theta}$ by a Taylor model with components

$T_{\theta_{i}}=\left(m\left(\Theta_{i}\right)+\left(\theta_{i}-m\left(\Theta_{i}\right)\right),[0,0]\right), \quad i=1, \cdots, p$.

Then, we can determine the Taylor model $\boldsymbol{T}_{\boldsymbol{f}^{[i]}}$ of the ITS coefficients $\boldsymbol{f}^{[i]}\left(\boldsymbol{y}_{j}, \boldsymbol{\theta}\right)$ by using RDA operations to compute $\boldsymbol{T}_{\boldsymbol{f}^{[i]}}=\boldsymbol{f}^{[i]}\left(\boldsymbol{T}_{\boldsymbol{y}_{j}}, \boldsymbol{T}_{\boldsymbol{\theta}}\right)$. The algorithmic procedure of phase 2 is summarized in Algorithm 1. The procedure begins with $\boldsymbol{V}_{0}=\mathbf{0}, \hat{\boldsymbol{T}}_{\boldsymbol{y}_{0}}=\left(m\left(\boldsymbol{Y}_{0}\right)+\right.$ $\left.\left(\boldsymbol{y}_{0}-m\left(\boldsymbol{Y}_{0}\right)\right),[0,0]\right)$, and $A_{0}=I . \quad J\left(\boldsymbol{f}^{[i]} ; \boldsymbol{Y}_{j}, \boldsymbol{\Theta}\right)$ denotes the interval extension of the Jacobian of $\boldsymbol{f}^{[i]}$ over $\boldsymbol{y}_{j} \in \boldsymbol{Y}_{j}$, and $\boldsymbol{\theta} \in \boldsymbol{\Theta}$. 
Table 1

Bioreactor microbial growth parameters

\begin{tabular}{llllll}
\hline Parameter & Value & Units & Parameter & Value & Units \\
\hline$\alpha$ & 0.5 & - & $\mu_{m}$ & {$[1.19,1.21]$} & day $^{-1}$ \\
$k$ & 10.53 & $\mathrm{~g} \mathrm{~S} / \mathrm{g} \mathrm{X}^{-1}$ & $K_{S}$ & {$[7.09,7.11]$} & $\mathrm{g} \mathrm{S} / 1$ \\
$D$ & 0.36 & day $^{-1}$ & $K_{I}$ & {$[0.49,0.51]$} & $(\mathrm{g} \mathrm{S} / 1)^{-1}$ \\
$S^{i}$ & 5.7 & $\mathrm{~g} \mathrm{~S} / 1$ & $X_{0}$ & {$[0.82,0.84]$} & $\mathrm{g} \mathrm{X} / 1$ \\
$S_{0}$ & 0.80 & $\mathrm{~g} \mathrm{~S} / 1$ & & & \\
\hline
\end{tabular}

\section{Numerical Experiments}

In a bioreactor, a simple microbial growth process [10], which involves a single biomass and single substrate, can be described using the following ODE model,

$$
\begin{aligned}
\dot{X} & =(\mu-\alpha D) X \\
\dot{S} & =D\left(S^{i}-S\right)-k \mu X,
\end{aligned}
$$

where $X$ and $S$ are concentrations of biomass and substrate, respectively; $\alpha$ is the process heterogeneity parameter; $D$ and $S^{i}$ are the dilution rate and the influent concentration of substrate, respectively; $k$ is the yield coefficient; and $\mu$ is the growth rate, which is dependent on $S$. We consider two models for $\mu$, the Monod law,

$\mu=\frac{\mu_{m} S}{K_{S}+S}$

and the Haldane law,

$\mu=\frac{\mu_{m} S}{K_{S}+S+K_{I} S^{2}}$

where $\mu_{m}$ is the maximum growth rate, $K_{S}$ is the saturation parameter, and $K_{I}$ is the inhibition parameter. In this study, the initial value of biomass concentration $X_{0}$, and the process kinetic parameters $\left(\mu_{m}, K_{S}\right.$, and $\left.K_{I}\right)$ are assumed to be uncertain and given by intervals. Thus, for the Monod law, there are three uncertain quantities, and four for the Haldane law. The values of the initial conditions $\left(X_{0}, S_{0}\right)$, the inputs $\left(D\right.$ and $\left.S^{i}\right)$, and parameters $\left(\alpha, k, \mu_{m}, K_{S}\right.$, and $\left.K_{I}\right)$ are given in Table 1.

We now report experimental results of a $\mathrm{C}++$ implementation of the method described above. This implementation is called VSPODE (Validating Solver for Parametric ODEs). The results for VSPODE were obtained using a $k=17$ order interval Taylor series method, and with a $q=5$ order Taylor model. For purposes of comparison, as a representative of traditional interval methods, we use the popular VNODE package [4], with a $k=17$ order interval Hermite-Obreschkoff QR method. Though, like other available solvers, VNODE is designed to deal with uncertain initial values, it can take interval parameter values as input. However, better performance can be obtained by treating the uncertain parameters as additional state variables with zero time derivatives; thus the parametric uncertainties become uncertainties in the initial values of the extra state variables. All tests were done using a constant step size of $h=0.1$, and were performed on a workstation running Linux with an Intel Pentium $43.2 \mathrm{GHz} \mathrm{CPU}$. 


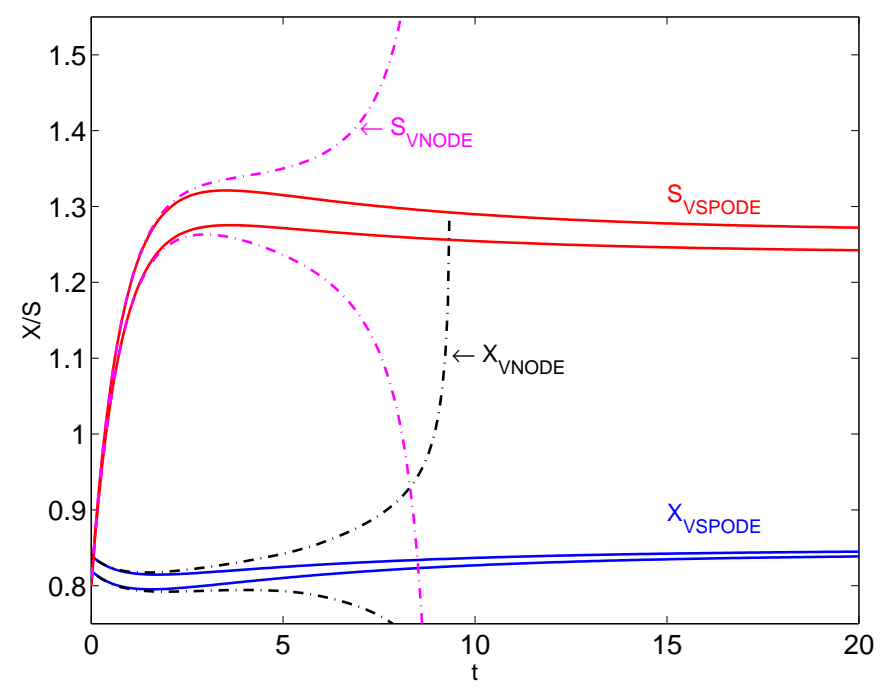

Figure 1. Enclosures for the Monod law

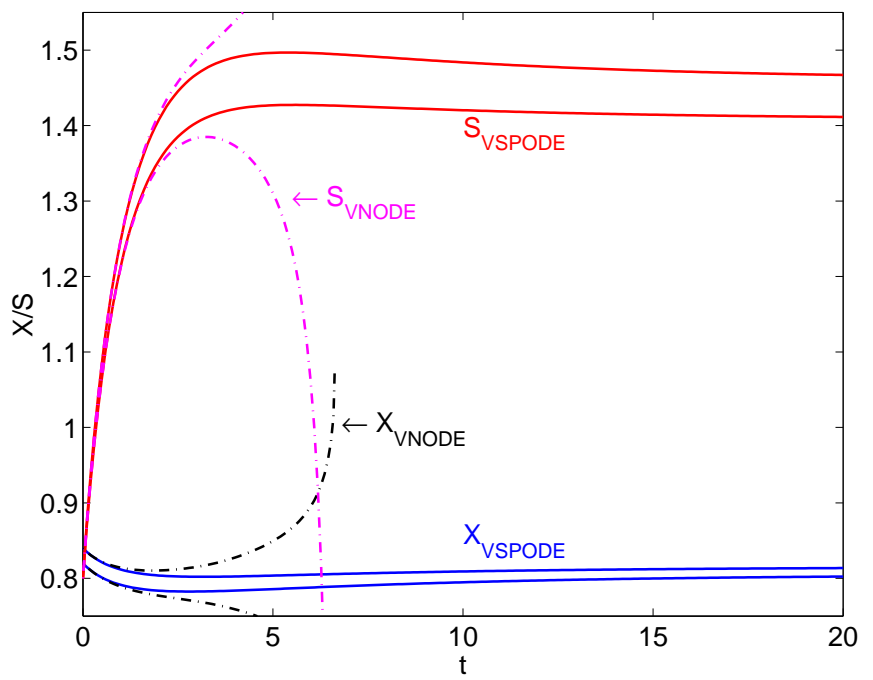

Figure 2. Enclosures for the Haldane law

The enclosures computed for $t \in[0,20]$ using VSPODE and VNODE for the Monod law and the Haldane law, are shown in Fig. 1 and Fig. 2, respectively. VSPODE clearly provides a better enclosure, with VNODE failing at $t=9.3$ for the Monod law, and at $t=6.6$ for the Haldane law. In order to allow VNODE to solve the problem all the way to $t_{m}=20$, we divided the intervals into a number of equal-sized sub-boxes and then used VNODE to determine the solution for each sub-box. The final solution enclosure is then the union of all the enclosures resulting from each sub-box. Results showing the final solution enclosures $\left(t_{m}=20\right)$ and their widths, as determined using VSPODE (with no box subdivision) and VNODE with an increasing number of sub-boxes, are given in Table 2 for the Monod law. For example, VNODE-1000 in Table 2 indicates the use of 1000 sub-boxes in VNODE. Even with 1000 sub-boxes, the solution enclosure determined by VNODE is still significantly wider than that obtained from a single calculation with VSPODE, and requires about 200 times more computation time. 
Table 2

Results for the Monod law, showing final enclosures $\left(t_{m}=20\right)$.

\begin{tabular}{lccc}
\hline Method & Enclosure & Width & CPU time $(\mathrm{s})$ \\
\hline VSPODE & {$[0.8386,0.8450]$} & 0.0064 & 1.34 \\
& {$[1.2423,1.2721]$} & 0.0298 & \\
\hline VNODE-343 & {$[0.8359,0.8561]$} & 0.0202 & 68.6 \\
& {$[1.2309,1.2814]$} & 0.0505 & \\
\hline VNODE-512 & {$[0.8375,0.8528]$} & 0.0153 & 102.8 \\
& {$[1.2331,1.2767]$} & 0.0436 & \\
\hline VNODE-1000 & {$[0.8380,0.8502]$} & 0.0122 & 263.1 \\
& {$[1.2359,1.2732]$} & 0.0373 & \\
\hline
\end{tabular}

\section{Concluding Remarks}

We have described a new method for obtaining validated solutions of initial value problems for ODEs with interval-valued initial conditions and parameters. The dependence of $\boldsymbol{y}^{\prime}(t)=\boldsymbol{f}(\boldsymbol{y}, \boldsymbol{\theta})$ on $t$ is handled using ITS methods, as in VNODE [4]. However, the dependence on the initial state $\boldsymbol{y}_{0}$ and the parameter vector $\boldsymbol{\theta}$ is handled by using, in a novel way, Taylor models of the form described by Makino and Berz [6,8]. Numerical results on a bioreactor kinetics problem demonstrate that this approach provides a very effective way to obtain an enclosure of all possible solutions to a parametric ODE system under uncertain conditions.

Acknowledgment. This work was supported in part by the State of Indiana 21st Century Research and Technology Fund and by the U. S. Department of Energy.

\section{References}

1. R. E. Moore, Interval Analysis, Prentice-Hall, Englewood Cliffs, NJ, 1966.

2. N. S. Nedialkov, K. R. Jackson and G. F. Corliss, Appl. Math. Comput., 105(1999), 21.

3. R. J. Lohner, Computations of guaranteed enclosures for the solutions of ordinary initial and boundary value problems, In Computational Ordinary Differential Equations, J. Cash and I. Gladwell (eds), Clarendon Press, Oxford, 1992.

4. N. S. Nedialkov, K. R. Jackson and J. D. Pryce, Reliab. Comput., 7(2001), 449.

5. M. Berz and K. Makino, Reliab. Comput. 4(1998), 361.

6. K. Makino and M. Berz, Remainder differential algebras and their applications, In Computational Differentiation: Techniques, Application, and Tools, M. Berz, C. Bishof, G. Corliss and A. Griewank(eds), SIAM, Philadelphia, 1996.

7. K. Makino and M. Berz, Reliab. Comput. 5(1999), 3.

8. K. Makino and M. Berz, Int. J. Pure Appl. Math., 4(2003), 379.

9. A. Neumaier, Reliab. Comput. 9(2002), 43.

10. G. Bastin and D. Dochain, On-line Estimation and Adaptive Control of Bioreactors, Elsevier, Amsterdam, 1990. 